\title{
IMPACT OF SOCIAL MEDIA ON THE FILM INDUSTRY
}

\begin{tabular}{|c|c|c|c|}
\hline \multicolumn{4}{|c|}{$\begin{array}{l}\text { Sachin Gupta } \\
\text { versity, Mathura, Uttar Pradesh, India }\end{array}$} \\
\hline Journal & \multicolumn{3}{|c|}{$\begin{array}{l}\text { Samvakti Journal of Research in Information Technology } \\
\text { https://www.sjrit.samvaktijournals.com } \\
\text { Volume } 2 \text { Year of Volume } 2021 \text { Page No : } 117 \text { - } 124\end{array}$} \\
\hline Discipline & \multicolumn{3}{|l|}{ Social Media } \\
\hline Conference & \multicolumn{3}{|c|}{$\begin{array}{l}\text { A virtual international conference on redefining and transforming the role of higher } \\
\text { education in sustainable development }\end{array}$} \\
\hline Conference & \multicolumn{3}{|c|}{ Start Date: September 30, 2021} \\
\hline Dates & \multicolumn{3}{|c|}{ End Date : September 30, 2021} \\
\hline Institute Name & \multicolumn{3}{|c|}{$\begin{array}{l}\text { JAIN (Deemed-to-be University) in association with Council for Industrial } \\
\text { Innovation and Research }\end{array}$} \\
\hline $\begin{array}{l}\text { Date Received } \\
\text { ID } \\
\text { Dol }\end{array}$ & $\begin{array}{l}\text { : November } 19,2021 \\
: 2021.02 .24 \\
: 10.46402 / 2021.02 .24\end{array}$ & $\begin{array}{l}\text { Publication Date } \\
\text { Paper Type } \\
\text { Dol URL }\end{array}$ & $\begin{array}{l}\text { : December 01, } 2021 \\
\text { : Conference Paper } \\
\text { : https://dx.doi.org/10.46402/2021.02.24 }\end{array}$ \\
\hline
\end{tabular}

\section{ABSTRACT}

Nowadays, most filmmakers are aggressively promoting films that utilize social media to broaden their audience's awareness of particular films. Only a few studies have looked at marketing campaigns, social media site activity, movie appraisal, and movie viewing preferences. The goal of this research was to determine how social media influenced the link among movie profiles and users. The goal of this study's research was to see whether social media might assist promote movies. A study was performed to find out what social media users were interested in, how they heard about movies, and whether or not a film's social media profile had any effect on them. The findings of this research may be utilized to create a social media marketing strategy for a production company. The goal of this research is to learn more about new media platforms and how to use them by looking at how social networking sites affect different elements of filmmaking.

KEYWORDS: Filmmaking, Filmmakers, Social Networking Sites, Social Media. 


\section{INTRODUCTION}

According to Wang et al., moviegoers like watching movies and often seek out information about them before going to see them, such as reading movie previews in magazines, browsing the internet, or asking friends or family members who really are movie stars. Potential viewers were also more eager to visit the picture in auditoriums after reading the film's social medias account on the official site. Audiences gain from Facebook and Twitter's continuing discussions with friends, as well as Twitter's encouragement of others to follow up on material. Tagg also invites viewers to engage with the movie more. Film reviews add to the enjoyment of viewing a movie and seeing the whole narrative unfold ${ }^{[1]}$. Figure 1 illustrates the eight Types of Social Media.

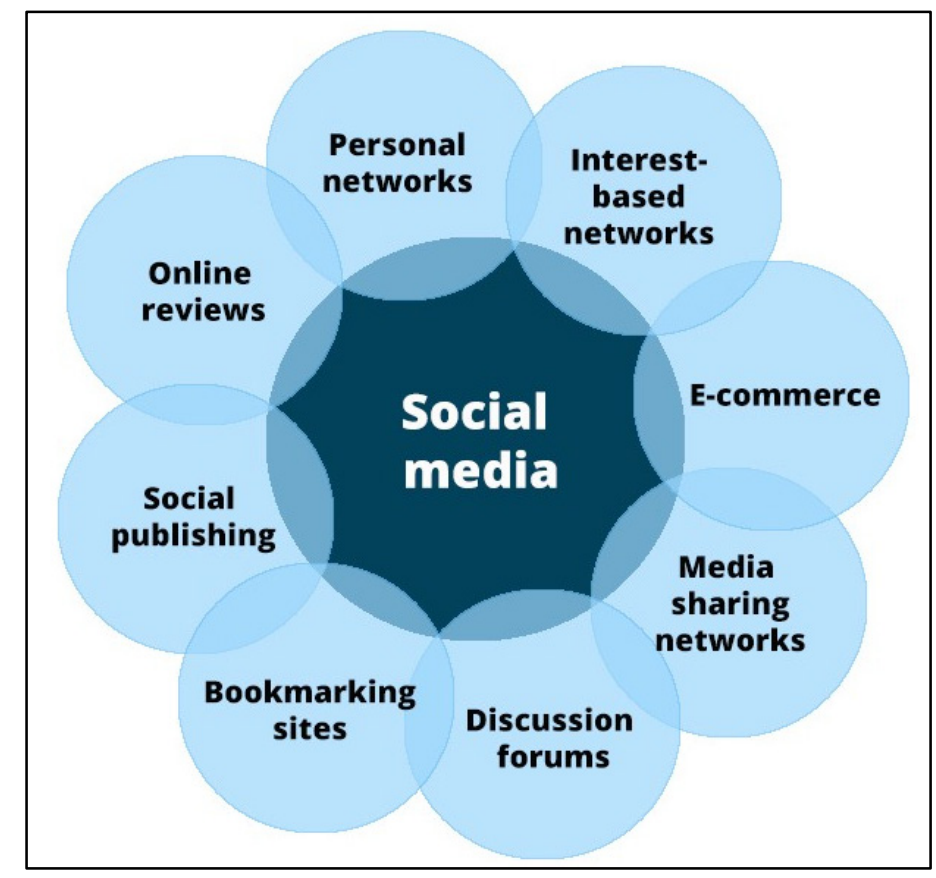

Figure 1: Illustrates the eight kinds of Social Media

These methods were utilized to instill confidence in the audience through eliciting emotions. After using the use as well as pleasure theories, the audiences believes that by analyzing the social as well as psychological features of others viewers, it is possible to discover and investigate the reasons for utilizing the media. The web not only shapes but also combines the borders of mainstream media and informal communication using the idea of use, this study investigates how Thai audiences choose to watch movies. In social and psychological roles, key reasons for using social media have been suggested, including cognitive requirements, social inclusive needs, individual needs for inclusions, as well as tension-related needs ${ }^{[2][3][4]}$. 
The present research examines two aspects: filmgoers' social media activities and the elements that impact the audience's decision-making process and subsequent watching of films in theaters; as well as the issues that affect the public's resolution making processes on what programs to see as movies. In this article, the investigator examines the character as well as influence of elements like advertising agendas, social media activity, as well as trailers reviews on the judgement to watch a movie in a theaters. In order to decide which variable is the most essential among them all, we must look at their mean differences ${ }^{[5]}$. Figure 2 illustrates the various kinds of social media apps.

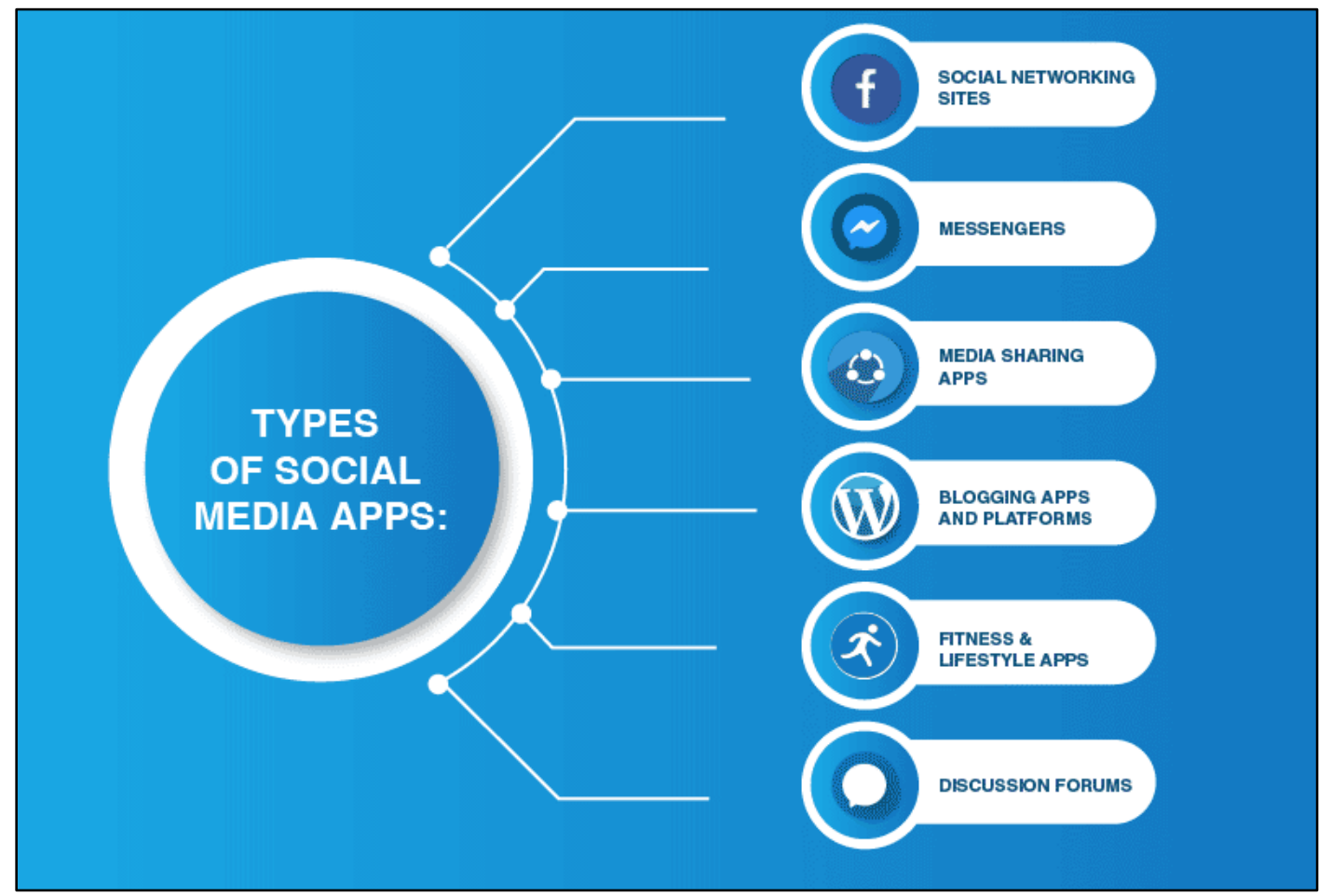

Figure 2: Illustrates the kinds of social media apps.

A multitude of factors determine whether a film is a box office hit or a flop in theaters. Some factors, such as the MPAA rating, film budget, and release date, are traditionally associated with the film, while others, such as the amount of likes on the film's Facebook page, the number of actors' Twitter followers, as well as the amount of likes on the film's trailers on YouTube, are socially generated promotions. We may investigate socially created Meta data acquired from socials Medias as well as multimedia places, as well as its impact on movie performance, in this case. The danger associated with creation any films has grown enormously as filmmakers struggle to match the public's escalating expectations. As a consequence, it's vital to comprehend the influences that influence the film's earnings. Preceding investigation has tried to resolve the issue of forecasting movie success or achievement, but it has done so in an inefficient manner by relying on traditional factors like the film script, advertisement spending plan, amount of opening 
cinema, production house, MPAA Rating, and star cast, among others ${ }^{[6]}$. Using data from Google as well as You Tubes trailer searches, critic appraisals on Twitter, and Wikipedia activity level, a numbers of fascinating lines of recents study have investigated the prerelease monetary movie achievement challenge. However, for movies with medium and low popularity, the models in performed considerably worse. We believe that the model's performance has deteriorated as a result of the above-mentioned works' selection of social media signal.As a result, identifying signals that are equally important to all movie profitability groups in order to develop categorization or prediction models is still a work in progress ${ }^{[7]}$.

According to Sood as well as Dreze a baptized title results in a higher promising ratings as well as desire to watch the movie. Instead of just shouting it Wall Street teo, the sequel was given the title "Wall St 2: Money Never Sleeps. It also serves as a refresher for people who haven't watched the prior installment in the series. It evaluate a sequel's success by the possibility of a sequel being made by the studio. According toauthors, named sequels which are not in the same genre as the first film had the best reviews were the most likely to be made. The scientists point out that the sequels investigated differed only little from their counterparts; if they differed greatly, the results may have been radically different. Companies in today's Hollywood are becoming more reliant on sequels to make money.

Recent studies have produced a mixed bag of findings, with some being remastered editions of Litman's work. Other study, such as Eliashberg et al. research, focuses on more contemporary problems, like the influence of socials media in making buzz for just a films or the uses of linear regression during the 'ecofriendly' phase. Brewer and others looked at the top hundred grossing films every year from 1999 - 2001 to see what factors went into anticipating box office success. Authors split their study into two sections. Extant estimates a film's performance founded on information's obtainable before to its release.

He found results that matched forecasts. The exalted regression indicated that budget plan, prequel gross, income, good critically reviews, as well as a release around the summertime or holiday period were all positively related with gross box office sales, which is consistent with prior research. The largest amount of screens, the star factor, film nominations, as well as word-of-mouth all had a favorable influence on gross box office earnings in the post facto regression. From 1997 through 2001, the top five box office hits had two tragedies, three comedies, one and activity picture, making it hard to determine which genre was dominating. 2. According to the study, personal wealth.

Simonton examines the impact of major accolades on a film's financial performance. He knows that not all Oscars are created equal when it comes to determining a movie's financial success, so he divides the many Oscars into four categories. The visual clusters includes photography, art direction, costuming, and makeup. The technical portion 
includes special effects, sound editing, including sound mixing, as well as a musical cluster. It's interesting to observe that a film's emotional elements garner greater critical acclaim, while the technical aspects earn a higher share of the budget. As a result, regardless of the fact that literary acclaim is correlated with a higher number of prizes, more money is spent on areas such as technological for the "wow" elements that draw in the audience. According to the author, a $\$ 1.00$ investment in technical 'wow' characteristics yields $\$ .47$ in return; a compromise between 'Film as Arts' and 'Film as Business.' A, critical acclaim isn't necessarily as correlated with higher box-office receipts as formerly assumed. In many occasions, he adds, a contender for such an award has been shown to bring in more income for a film than an awards show triumph.

\section{DIscussion}

The selection of a film may be influenced by a number of things. Before choosing a film, consumers consider the marketing message, the film's unbiased source of information, the film's features, the film's content, and the film's accessibility Customers that have seen or have been introduced to something like a video in marketing and communications tell their friends about it, resulting in increased term communication via direct encounters or social media sites. Movie critics commonly post reviews on the internet, in magazines, and in newspapers, which contain previews and a storyline summary. As a consequence, buyers can evaluate the historical, horrific, and technological content in films. When a spectator can compare a trailer's want to see a given movie with the desire to see another movie, the audience is more likely to be "sold" on the marketed film. Other media's trailer trails, which are tied to the audience's emotions and expectations, will likewise pique people's attention and draw them in.

We're trying to figure out how the social networking signals we've gathered affect movie profitability classification. We categorize movies into three groups depending on their profitability to determine how well they perform. We examine the outcomes of well-known deep learning support - vector Networks, Packaged Decision Tree, Adaptive Boosted Logistic Regression, Randomized Forest, and Decision Tree - to determine which strategy is best for our data and issue situation. Previous research using a similar algorithms for classification difficulties inspired the machine learning methodologies we used. A second comparison study is also carried out to evaluate whether social networking signal is the most reliable predictor of movie revenue. We utilize F-Score values to indicate the accuracy of a tests since categorization of our kind is believed to be have extreme accuracy but low recall. To better depict the accuracy of our tests, we used F-Score. 
F-Score $=(2 *$ precision $*$ recall $) /($ precision recall $)$ is the harmonic mean of precision and recall.

Equation 1 : F-Score formula used to depict accuracy

The purpose of this research was to see how successful it was to promote a movie using social media. The majority of movies nowadays use social media to boost their marketing efforts. New methods using contemporary social media, on the other hand, may be developed to enhance audience/studio interactions and film recognition. Our findings show that social media has a significant influence on movie success and gives additional information on movie profitability. We don't propose a novel machine learning technique in this study; instead, we utilize carefully selected social media data to assess the performance of really good methods in the research environment. We demonstrate that an actor's popularity among Twitter users is the single most important factor inside a film's box office hit. Another intriguing finding is that Facebook likes cannot be relied upon as a reliable indicator in comparable analyses.

Our findings indicate that the like signal contains noise, which limits its capacity to be analyzed. Finally, we can observe that Support Vector Machine, a contemporary classification method, outperformed previous classification techniques. This discovery paves the way for future academics to dig further into and possibly utilize other aspects of the facts that Twitter followers count is a reliable indicator of popularity. It may be useful to gauge popularity by looking at the number of followers for other elements of the film, such as the production studio or the director. Furthermore, our findings suggest that Twitter may be a useful tool for gauging popularity. Future scholars are encouraged to use Twitter's welcome page to find out how many fans and followers a film has. We anticipate the data to become cleaner and more trustworthy in the future, thanks to ongoing research into building classifications algorithms to distinguish between bots, false accounts, as well as people on Twitter. Also, although Facebook likes did not show to be a reliable predictor of movie profitability, we think that Facebook's recommendation power may be further explored utilizing the OpenGraph Protocol.

\section{CONCLUSION}

The goal of this research was to look at how social media marketing impacted the connection between users (audience) and film profiles (production studios). Because most individuals use social media to communicate, using it to create connections between audience members, films, and production companies may be beneficial. The purpose of social media is to provide information that allows viewers to have a more in-depth look at various elements of the film. Images, articles, promos, and behind-the-scenes material are all examples of this. Even while individuals were on social media and did hear about movies via it, the study found that they did not actually engage with movie profiles created by production companies. 
The majority of respondents indicated they were most likely to hear about movies via social media, indicating that this medium has an effect on moviegoers. Family and friends were the second most common way for participants to learn about movies. Because family and friends, as well as social media, accounted for over $70 \%$ of people's first and second choices, it's safe to assume that these are the most popular methods to learn about movies. Furthermore, the majority of participants (54.59 percent) visited these channels on occasion to learn more about the movie and the material that accompanied it. People were mainly searching for movies, photos, or behind-the-scenes information in these networks. Reviews, trailers, and information on the actors in the film were also recommended as additional material. This indicates that if these profiles had more varied material in addition to what they currently provide, individuals would be more interested in visiting them. Overall, a link between moviegoers, filmmakers, and production companies has been established. Studio executives will be able to adopt new marketing tactics based on the findings, which will include utilizing social media to engage with viewers. 


\section{REFERENCES:}

[1] Shruti, S. D. Roy, and W. Zeng, "Influence of social media on performance of movies," 2014, doi: 10.1109/ICMEW.2014.6890664.

[2] Y. Liang and W. Shen, "Fan economy in the Chinese media and entertainment industry: How feedback from super fans can propel creative industries' revenue," Glob. Media China, 2016, doi: 10.1177/2059436417695279.

[3] H. Bathelt and A. Gräf, "Internal and external dynamics of the Munich film and TV industry cluster, and limitations to future growth," Environ. Plan. A, 2008, doi: 10.1068/a39391.

[4] B. Karlin and J. Johnson, "Measuring Impact: The Importance of Evaluation for Documentary Film Campaigns," M/C J., 2011, doi: 10.5204/mcj.444.

[5] K. Baker, "Social Media Marketing in the Film Industry: Using Social Media to Create a Successful Marketing Campaign for the Theatrical Release of a Film," Hilos Tensados, 2016.

[6] M. Pütter, "The Impact of Social Media on Consumer Buying Intention," J. Int. Bus. Res. Mark., 2017, doi: 10.18775/jibrm.1849-8558.2015.31.3001.

[7] O. O. Fatoki, "The Impact of Human, Social and Financial Capital on the Performance of Small and Medium-Sized Enterprises (SMEs) in South Africa," J. Soc. Sci., 2011, doi: 10.1080/09718923.2011.11892970.

[8] C. M. Pulido, G. Redondo-Sama, T. Sordé-Martí, and R. Flecha, "Social impact in social media: A new method to evaluate the social impact of research," PLoS One, 2018, doi: 10.1371/journal.pone.0203117.

[9] F. P. Tajudeen, N. I. Jaafar, and S. Ainin, "Understanding the impact of social media usage among organizations,” Inf. Manag., 2018, doi: 10.1016/j.im.2017.08.004.

[10] C. Drummond, H. McGrath, and T. O'Toole, "The impact of social media on resource mobilisation in entrepreneurial firms," Ind. Mark. Manag., 2018, doi: 10.1016/j.indmarman.2017.05.009.

END 